\title{
Correction to: Desformylflustrabromine, a positive allosteric modulator of $\alpha 4 \beta 2$-containing nicotinic acetylcholine receptors, enhances cognition in rats
}

\author{
Agnieszka Nikiforuk $^{1} \cdot$ Ewa Litwa $^{1} \cdot$ Martyna Krawczyk ${ }^{1} \cdot$ Piotr Popik $^{1} \cdot$ Hugo Arias $^{2}$ \\ Published online: 8 July 2020 \\ (c) Maj Institute of Pharmacology Polish Academy of Sciences 2020
}

Correction to: Pharmacological Reports (2020) 72:589-599 https://doi.org/10.1007/s43440-020-00092-4

In the Acknowledgements section of this article the incorrect information was included inadvertently.

The correct Acknowledgements should read as follows:

Acknowledgements This study was supported by the Statutory Activity of the Maj Institute of Pharmacology, Polish Academy of Sciences, Kraków, Poland and by the Polish National Science Centre (NCN) Grant no. 2016/23/B/ NZ7/01131.

The original article can be found online at https://doi.org/10.1007/ s43440-020-00092-4.

Agnieszka Nikiforuk

nikifor@if-pan.krakow.pl

1 Department of Behavioral Neuroscience and Drug Development, Maj Institute of Pharmacology Polish Academy of Sciences, 12 Smetna Street, 31-343 Krakow, Poland

2 Department of Pharmacology and Physiology, Oklahoma State University College of Osteopathic Medicine, Tahlequah, OK, USA 